\title{
La prospective en éducation
}

Federica Minichiello

\section{OpenEdition}

Journals

Édition électronique

URL : https://journals.openedition.org/ries/4396

DOI : $10.4000 /$ ries.4396

ISSN : 2261-4265

\section{Éditeur}

France Education international

\section{Édition imprimée}

Date de publication : 1 septembre 2015

Pagination : 12-14

ISSN : 1254-4590

\section{Référence électronique}

Federica Minichiello, «La prospective en éducation », Revue internationale d'éducation de Sèvres [En ligne], 69 | septembre 2015, mis en ligne le 01 septembre 2015, consulté le 01 juillet 2021. URL : http:// journals.openedition.org/ries/4396; DOI : https://doi.org/10.4000/ries.4396

Ce document a été généré automatiquement le 1 juillet 2021

(c) Tous droits réservés 


\title{
La prospective en éducation
}

\author{
Federica Minichiello
}

1 Le futur de l'éducation soulève de nombreux questionnements. Ces dernières années ont été caractérisées par un engouement autour des MOOCs ainsi que par l'accès à des données massives et à leur utilisation dans le domaine des sciences cognitives et de l'apprentissage personnalisé. Face aux interrogations sur les évolutions des pratiques et le rôle des acteurs en éducation, la prospective offre un aperçu du périmètre du possible.

2 Un regard prospectif dépasse les schémas de pensée du présent pour imaginer des histoires alternatives du futur; l'approche est créative : on définit des représentations possibles de l'avenir, caractérisées par des degrés variables de souhaitabilité, en précisant ainsi les marges de manœuvre dont disposeraient les décisionnaires politiques. L'ambition est de décrire des réalités inexpérimentées, sans pour autant dériver dans l'utopie.

3 L'exercice prospectif en éducation est complexe en raison de deux facteurs : le nombre important d'acteurs et d'éléments dans l'écosystème (enseignants, élèves, infrastructures, technologies, marché de l'emploi, démographie etc.) et l'horizon temporel particulièrement étendu à étudier.

4 Le présent article propose quelques éléments descriptifs de la prospective et une sélection de travaux en éducation disponibles en ligne. Les tendances présentées sont seulement esquissées: dans la publication de l'OCDE «Repenser l'enseignement, des scénarios pour agir ", on définit un scénario comme volontairement court, schématique, afin de « laisser à dessein beaucoup de choses à imaginer $»^{1}$.

\section{Comprendre la prospective}

\section{European Foresight Plateform (EFP)}

5 European Foresight Plateform est un réseau qui vise à mobiliser les différents acteurs de la prospective en Europe. Il propose notamment "For-Learn", une plateforme pédagogique lancée lors du sixième programme-cadre de la Commission européenne 
(2002-2006), recensant de nombreuses ressources sur la prospective, ses différentes méthodologies et une grille comparative des techniques les plus utilisées, comme la construction de scénarios, les méthodes d'enquête Delphi' ${ }^{2}$, l'analyse SWOT (forcesfaiblesses-opportunités-menaces) etc. [http://www.foresight-platform.eu]

\section{World Future Studies Foundation (WFSF)}

Cette ONG internationale, partenaire des Nations unies et plus particulièrement de l'Unesco, réunit chercheurs, professionnels, étudiants et institutions qui s'intéressent aux études futuristes. Parmi ses initiatives on peut citer notamment la conférence annuelle World Conference of Futures Research et le laboratoire pédagogique LEALA : un environnement d'apprentissage en ligne proposant une sélection très diversifiée de ressources pour découvrir la prospective, un répertoire de formations dans le domaine et plusieurs documents vidéo, comme par exemple une intervention de Riel Miller, qui dirige la prospective à l'Unesco, sur «la discipline de l'anticipation». Selon lui, la prospective n'est pas une perception fondée sur une logique du temps présent, ni une planification sur la base d'un objectif déjà fixé, mais une forme d'intelligence collective qui analyse la réalité, les éléments récurrents et les points de divergences et anticipe des évolutions, indépendamment de ce que l'on souhaiterait. [http://www.wfsf.org]

\section{Sélection de travaux de prospective en éducation}

\section{Publications institutionnelles}

\section{OCDE/CERI. L'école de demain}

7 Le programme "L'école de demain», mené par le Centre pour la recherche et l'innovation dans l'enseignement (CERI), a fortement lancé la réflexion prospective en éducation. Les six scénarios élaborés en 2001 concernaient les 15-20 ans à venir et restent une référence en matière d'évolutions de l'école : une situation de statu quo (le maintien d'un système institutionnalisé, qui s'oppose radicalement au changement, ou l'extension de la logique de marché à l'école); une possible "re-scolarisation " (le renforcement de l'autonomie des écoles et de leur rôle pivot dans la société ou les écoles comme organisations apprenantes, souples, avec des programmes personnalisés); l'hypothèse de la "déscolarisation" (la naissance de réseaux d'apprentissage à grande échelle ou la fin du modèle, la désintégration du système éducatif). [http://www.oecd.org/fr/sites/educeri/ceri-lecolededemain.htm]

\section{Commission européenne. Institute for Prospective Technological Studies}

Le JRC-IPTS est l'un des sept instituts du Joint Research Center, le laboratoire de recherche scientifique et technique de l'Union européenne. Parmi leurs travaux en éducation, on peut citer notamment « Mapping Major Changes to Education and Training in 2025 », un travail de classification de plus de deux cent idées de changement par degré d'importance et de faisabilité. Parmi les évolutions les plus notables figurent la personnalisation des contenus et la nature sociale de l'apprentissage, la généralisation des ressources libres étant considérée comme l'innovation la plus plausible. Le thème de l'éducation «ouverte » a été repris dans Open Education 2030, une publication issue 
d'un appel à communications sur les scénarios éducatifs d'ici 2030: parmi les tendances, on retrouve la personnalisation, la généralisation des environnements d'apprentissage comme le « Self Organising Learning Environment (SOLE) 3 » et l'émergence d'espaces en ligne individuels où retrouver l'information et les données nécessaires à l'apprentissage, à l'évaluation et à la certification. https://ec.europa.eu/jrc/en/ institutes/ipts

\section{Des revues prospectives}

\section{European Journal of Futures Research}

Cette revue scientifique en open access, lancée en 2013, est consacrée à la prospective et aux études futuristes, dans une approche comparative. Parmi les sujets récemment traités en éducation: le futur de l'éducation en Europe et l'innovation dans l'enseignement supérieur en 2025. Les scénarios élaborés imaginent, par exemple, une «explosion» des MOOCs, une publication scientifique presque instantanée avec une validation par les pairs a posteriori, la création de grands pôles universitaires régionaux, l'émergence d'un nouvel emploi de «relations publiques» dans les universités, qui facilite le dialogue entre recherche et entreprises. [http://link.springer.com/journal/ 40309]

\section{Futuribles}

10 Centre français d'études et de réflexion, Futuribles publie une revue homonyme, qui a traité de sujets comme l'école en 2030, la profession enseignante en 2020 , etc. ${ }^{4}$ Son étude " pour l'enseignement et la formation à l'horizon 2025 » propose une série de notes thématiques sur l'avancée des sciences cognitives, la compétition et la coopération internationale dans l'enseignement supérieur et un catalogue de 85 innovations pédagogiques, comme le programme pilote néerlandais Dream School, un projet collaboratif de boîte à suggestions pour illustrer les caractéristiques de « l'école rêvée ». [https://www.futuribles.com] [http://projectdreamschool.org]

\section{Think thanks et initiatives collaboratives}

\section{Millennium Project}

11 Ce think thank a été fondé en 1996, après une étude de faisabilité de trois ans menée en particulier par l'Université des Nations unies (http://unu.edu). Il publie le rapport annuel «État du futur ", dont la dernière édition (2013-2014) préconise de nouvelles formes de travail individuel, en opposition au modèle actuel de travail local ainsi qu'une base de données mondiale recensant tous les plans de stratégie politique à long terme. Dans la partie sur l'éducation et l'apprentissage d'ici 2030, on constate plusieurs tendances comme l'apprentissage en temps réel, l'éducation individualisée, la simulation virtuelle et la réalité augmentée, une connaissance accrue des mécanismes cérébraux d'apprentissage. Des interrogations émergent: les méthodes de répartition sociale de ces nouvelles possibilités d'intelligence, au risque de creuser ultérieurement les inégalités et une frontière de moins en moins distincte entre mondes virtuel et réel. [http://millennium-project.org] 


\section{Institute for the future (IFTF)}

12 L'Institute for the future est un organisme de recherche à but non lucratif localisé dans la Silicon Valley. Son approche très particulière met l'accent sur les pratiques collaboratives et la ludification. Toute personne peut engager des conversations sur le futur, dans un format rigoureusement succinct, et proposer des idées, gagner des points lorsque d'autres adhèrent au projet, et co-construire des scénarios dans des laps de temps très courts (24-72 heures maximum).

Son programme en éducation «Future of Learning " choisit comme champ d'étude les apprenants « extrêmes », souvent à la marge des systèmes éducatifs, et qui construisent leur éducation en puisant dans les ressources libres et la formation en ligne. L'idée est d'étudier des individus qui maîtrisent des techniques d'apprentissage pour induire des progrès importants dans les neurosciences. L'apprentissage deviendrait une expérience quotidienne, dissociée d'un environnement physique, grâce à un flux continu de ressources, des mentors disponibles en temps réel et de nouvelles méthodes de certification privilégiant la mise en situation. [http://www.iftf.org]

\section{Global Education Futures Forum}

14 Cette plateforme collaborative sur les transformations souhaitables dans l'écosystème éducatif est portée par la Fondation russe Skolkovo et le Massachusetts Institute of Technology (MIT). Le rapport Education Future Global Agenda suggère des idées particulièrement extrêmes: des universités d'un milliard d'étudiants, des instances comme le «World Education and Talent Organisation» pour régir la mobilité des talents et définir des standards internationaux ou un protocole de Kyoto "spécial capital humain", qui établirait des règles de compensation entre nations, pour équilibrer les écarts en termes d'investissement en éducation et la fuite des cerveaux. D'ici 2030, le jeu deviendrait l'activité prédominante de l'homme. Les élèves se rapproprieraient leurs données d'apprentissage et de nouveaux modèles apparaîtraient, comme l'investissement direct sur les talents ${ }^{5}$.

[http://edu2035.org]

15 L'exercice prospectif en éducation s'insère dans une réflexion sur les évolutions de la société dans sa globalité : une société de demain dont les caractéristiques demeurent inconnues. Dans le domaine de la recherche et développement, le projet européen VERA (Forward Visions on the European Research Area) propose ainsi plusieurs scénarios prospectifs de la vie en 2030: un contexte de raréfaction ou de disparition de ressources, une société civile qui s'empare des défis sociétaux, dans laquelle la coopération et la mobilité transnationale seraient une réalité. Les citoyens produiront leur énergie, imprimeront en 3D leurs biens de consommation et deviendront acteurs de leur éducation, de leur travail, d'une « science des citoyens ». [http://eravisions.eu] 


\section{NOTES}

1. J. Ogilvy, L'enseignement à l'ère de l'information: scénarios, équité et égalité, OCDE (2006).

2. Méthode d'enquête qui permet de structurer la réflexion d'un groupe d'experts, par un cycle de questions-réponses gérées dans l'anonymat, qui se termine lorsqu'un niveau de consensus acceptable est obtenu sur les questions posées. (Source : UNIGE)

3. Environnement d'apprentissage auto-organisé, imaginé par le professeur Sugata Mitra. Pour plus d'informations, se référer au projet School in the Cloud [ https:// www.theschoolinthecloud.org].

4. A. Bouvier, «Préparons l'école de 2030 », Futuribles, 09/2012, n³8; F. Cros, "Quels enseignants en 2020 ? », Futuribles, 09/2001, n 267.

5. Cette tendance est déjà d'actualité, comme le montre la plateforme de financement participatif Upstart [https://www.upstart.com].

\section{INDEX}

Mots-clés : prospective en éducation, scénarios prospectifs

Keywords : prospective scenarios, prospective on education

Palabras claves : prospectiva en educación, escenarios prospectivos

\section{AUTEUR}

\section{FEDERICA MINICHIELLO}

Federica Minichiello a fait des études d'économie à l'Université de Tor Vergata (Italie) et d'ingénierie documentaire au Conservatoire national des arts et métiers (CNAM) de Paris. Elle est chargée de veille au centre de ressources et d'ingénierie documentaires du CIEP. Également rédactrice pour Thot Cursus, elle s'intéresse en particulier aux évolutions des pratiques et des politiques éducatives, en lien avec le numérique. minichiello@ciep.fr 